\title{
Prognostic value of echocardiography and ECG in heart failure with preserved ejection fraction
}

\author{
Cenkerova $\mathrm{K}^{1}$, Dubrava $\mathrm{J}^{2}$, Pokorna $\mathrm{V}^{3}$, Kaluzay $\mathrm{J}^{3}$, Jurkovicova $\mathrm{O}^{3}$ \\ Department of General Cardiology, National Institute of Cardiovascular Diseases, Bratislava, Slovakia. \\ kmardiakova@gmail.com
}

\section{ABSTRACT}

OBJECTIVE: The aim of this study was to evaluate characteristics of patients with heart failure (HF) with preserved ejection fraction (HFPEF) and to assess prognostic predictors in 2-year follow-up.

METHODS: We included prospectively 109 patients admitted to the internal department for HF, grouped into HFPEF $(E F>40 \%, n=63)$ and HF with reduced EF (HFREF) $(E F \leq 40 \%, n=46)$. Preserved right ventricular systolic function (PRV) was defined as the peak systolic tricuspid annular velocity (S') $>10.8 \mathrm{~cm} / \mathrm{s}$.

RESULTS: HFPEF and HFREF patients had non-significantly different 2-year all-cause and CV mortality $(28.6 \%$ vs $37.0 \%, 17.5 \%$ vs $21.7 \%)$. Patients with HFPEF and PRV vs dysfunctional RV had a better survival $(76.6 \%$ vs $56.3 \%, p=0.045)$. In HFPEF, the patients who survived had a trend to better $S^{\prime}(13.6 \pm 3.1 \mathrm{~cm} / \mathrm{s}$ vs $11.9 \pm 3.4$ $\mathrm{cm} / \mathrm{s}, p=0.055)$, shorter QTc $(427 \pm 42 \mathrm{~ms}$ vs $454 \pm 42 \mathrm{~ms}, p=0.058)$, and all-cause mortality was lowered only by anticoagulants $(12.0 \%$ vs $39.5 \%, p=0.02)$. QTc interval and PRV emerged as predictors of all-cause mortality (HR 1.7 per 40 ms change, $95 \% \mathrm{Cl} 1.1-2.6, p=0.02$, HR 0.38, $95 \% \mathrm{Cl} 0.15-0.93, p=0.03$ ).

CONCLUSIONS: In HFPEF, we observed a trend to lower all-cause and CV mortality compared to HFREF and anticoagulants were the only therapy that significantly lowered mortality. PRV and QTc interval emerged as independent predictors of survival (Tab. 6, Fig. 2, Ref. 26). Text in PDF www.elis.sk.

KEY WORDS: HFPEF, prognosis, QTc, right ventricular function.

Limitations: The results of our study are limited by the relatively small groups of patients with both HFPEF and HFREF and by the mid-term follow-up period. The assessment of the RV systolic function was limited to just one parameter - the peak systolic velocity of the lateral tricuspid annulus in tissue Doppler imaging.

\section{Introduction}

Heart failure (HF) with preserved left ventricular ejection fraction (HFPEF) is a common form of HF in the population (1). HFPEF comprises almost $50 \%$ of HF patients, depending on definition (2-4). The prognosis is generally poor, and it remains unclear whether it is better or worse compared to heart failure with reduced left ventricular ejection fraction (HFREF) $(2,5-8)$. The immediate post-discharge prognosis is better in HFPEF but longterm mortality is similar $(2,9)$. Annual mortality in HFPEF ranges from $10-30 \%$, with around $60 \%$ of cardiovascular (CV) deaths, which comprises mostly sudden death and HF death depending

${ }^{1}$ Department of General Cardiology, National Institute of Cardiovascular Diseases, Bratislava, Slovakia, ${ }^{2}$ Department of Noninvasive Cardiology, Ss. Cyril and Methodius Hospital, University Hospital, Bratislava, Slovakia, and ${ }^{3} 4$ th Department of Internal Medicine, Ss. Cyril and Methodius Hospital, University Hospital, Bratislava, Slovakia

Address for correspondence: K. Cenkerová, Department of General Cardiology, National Institute of Cardiovascular Diseases, Pod Krasnou horkou 1, SK-83348, Bratislava, Slovakia.

Phone: +421.2.59320442, Fax: +421.2.54788745 on the clinical or epidemiological type of study $(2,9,10)$. Even though many studies were performed with the focus on prognosis improvement, no effective treatment was found (11).

We set this study to determine the complex characteristics of HFPEF, its prognosis and the possible predictors of survival in patients routinely treated in the internal department.

\section{Methods}

We prospectively enrolled 109 consecutive patients with HF admitted to the Department of Internal Medicine within a one-year period (2010-2011). Patients with known advanced malignant tumors, whose expected survival was less than one year, were excluded. The diagnosis of HF was made according to the guidelines of the European Society of Cardiology (11). Patients were divided into the two groups according to the left ventricular ejection fraction (LVEF), as assessed by echocardiography: HFPEF with LVEF $>40 \%(n=63)$ and HFREF with LVEF $\leq 40 \%(n=46)(2,9)$.

The vital status of the patients was ascertained at regular visits, by general practitioners, and from hospital records as well as by family members. All-cause mortality was defined as death from any cause and $\mathrm{CV}$ mortality as death from any documented $\mathrm{CV}$ cause. Cumulative CV endpoint was defined as all-cause mortality or myocardial infarction or stroke. Patients were followed-up for 24 months and visits were scheduled every 6 months after discharge. All echocardiographic measurements were performed according to the American Society of Echocardiography (12). LV 
407-412

diastolic dysfunction was classified into the three degrees (13). We calculated the LVEF according to the Simpson's biplane method. Right ventricular (RV) systolic function was evaluated by the peak systolic velocity of the lateral tricuspid annulus (S') in tissue Doppler imaging. Normal RV systolic function was defined as the $S$ ' value more than $10.8 \mathrm{~cm} / \mathrm{s}(13,14)$. Pulmonary artery systolic pressure (sPAP) was calculated as the maximal gradient of tricuspid regurgitation + the estimated value of the right atrial systolic pressure. We measured the vena cava size as the maximum diameter of vena cava before merging with the right atrium during expirium. The study was performed according to the Declaration of Helsinki and hospital ethical standards.

\section{Statistical analysis}

Categorical data were expressed as frequencies and percentages. Continuous data were expressed as the means and standard deviations and in case of abnormal distribution as the median and interquartile range (IQR). The comparison of categorical variables was performed using the Fisher's exact test. The comparison of continuous variables was performed using T-test and Mann-Whitney non-parametric U-test. Correlations were analyzed with a linear regression analysis and non-parametric Spearman's correlation coefficient. The log-rank test was used to determine if survival was significantly different. Hazard ratios (HRs) were calculated using Cox proportional hazards model. We included all examined parameters (demographic data, therapy, echocardiographic, ECG and laboratory parameters) in the hazard ratio models and in statistical analyses of mortality data. Statistical significance was defined as $p<0.05$. Data were analyzed using the statistical program JMP7, SAS, USA.

\section{Results}

Table 1 shows basic demographic characteristics on admission. Patients with HFPEF were significantly older with a higher prevalence of female gender and arterial hypertension as compared to HFREF. In the 2-year follow-up, there was a substantial increase in the prevalence of atrial fibrillation in HFPEF and not so prominent increase in HFREF $(78 \%$ vs $48 \%, p=0.012)$. We observed a decrease in the mean systolic blood pressure during final visit compared to the baseline in both HFPEF and HFREF

Tab. 1. Basic demographic parameters on admission.

\begin{tabular}{lccc}
\hline & $\begin{array}{c}\text { HFREF } \\
(\mathrm{n}=46)\end{array}$ & $\begin{array}{c}\text { HFPEF } \\
(\mathrm{n}=63)\end{array}$ & $\mathrm{p}$ \\
\hline Male, \% & 76.1 & 54.0 & $<0.05$ \\
Age, years & $66.8 \pm 12.1$ & $74.1 \pm 9.8$ & $<0.01$ \\
Body mass index, kg/m2 & $27.6 \pm 4.3$ & $30.3 \pm 6.5$ & $<0.01$ \\
Arterial hypertension, \% & 73.9 & 90.5 & $<0.05$ \\
Diabetes mellitus, \% & 34.8 & 41.3 & $\mathrm{NS}$ \\
Coronary artery disease, \% & 69.6 & 68.3 & $\mathrm{NS}$ \\
History of atrial fibrillation, \% & 28.6 & 47.5 & 0.06 \\
Systolic blood pressure, mmHg & $125(110-145)$ & $145(130-160)$ & $<0.001$ \\
Diastolic blood pressure, mmHg & $80(70-85)$ & $80(70-90)$ & $\mathrm{NS}$ \\
\hline HFPEF - heart failure with preserved ejection fraction, HFREF - heart failure with \\
reduced ejection fraction, NS - non-significant & \multicolumn{3}{l}{}
\end{tabular}

Tab. 2. Baseline echocardiographic parameters.

\begin{tabular}{lccc}
\hline & $\begin{array}{c}\text { HFREF } \\
(\mathrm{n}=46)\end{array}$ & $\begin{array}{c}\text { HFPEF } \\
(\mathrm{n}=63)\end{array}$ & $\mathrm{p}$ \\
\hline LVEF, \% & $26.6 \pm 8.2$ & $59.0 \pm 8.7$ & $<0.001$ \\
LVEDD, mm & $61.9 \pm 8.1$ & $49.2 \pm 5.9$ & $<0.001$ \\
LVMI, g/m2 & $143(119-193)$ & $135(106-166)$ & $\mathrm{NS}$ \\
$\mathrm{LAD}, \mathrm{mm}$ & $53.4 \pm 5.9$ & $49.7 \pm 4.7$ & $<0.001$ \\
$\mathrm{E}, \mathrm{m} / \mathrm{s}$ & $1.05 \pm 0.37$ & $1.15 \pm 0.35$ & $\mathrm{NS}$ \\
$\mathrm{A}, \mathrm{m} / \mathrm{s}$ & $0.73(0.45-0.93)$ & $0.77(0.60-0.99)$ & $<0.01$ \\
$\mathrm{E} / \mathrm{A}$ & $2.21(1.31-3.17)$ & $1.31(0.78-1.83)$ & $<0.05$ \\
DCT, ms & $127(105-145)$ & $158(137-193)$ & $<0.001$ \\
IVRT, ms & $55(42-68)$ & $50(44-70)$ & $\mathrm{NS}$ \\
$\mathrm{E}^{\prime}, \mathrm{m} / \mathrm{s}$ & $0.08(0.06-0.09)$ & $0.10(0.07-0.13)$ & $<0.05$ \\
E/E' & $12.3(9.6-16.4)$ & $11.4(8.1-16.1)$ & $\mathrm{NS}$ \\
Diastolic dysfunction grade $^{\prime}$ & $3(2-3)$ & $2(1-3)$ & $<0.01$ \\
$\mathrm{~S}^{\prime}, \mathrm{cm} / \mathrm{s}$ & $10.7 \pm 3.7$ & $13.1 \pm 3.2$ & $<0.001$ \\
Preserved RV systolic function, $\%$ & 41.3 & 74.6 & $<0.001$ \\
sPAP, mmHg* & $44.3 \pm 11.2$ & $46.8 \pm 12.8$ & $\mathrm{NS}$ \\
\hline
\end{tabular}

*patients with adequately measurable jet of tricuspid regurgitation, A - late mitral filling velocity, DCT - E wave deceleration time, E - peak early mitral filling velocity, $E^{\prime}-$ peak early diastolic mitral annular velocity, HFPEF - heart failure with preserved ejection fraction, HFREF - heart failure with reduced ejection fraction, IVRT - isovolumic relaxation time, LAD - left atrial diameter in parasternal long axis, LVEF - left ventricular ejection fraction, LVEDD - left ventricular end-diastolic diameter, LVMI - left ventricular mass index, NS - non-significant, RV - right ventricular, $\mathrm{S}^{\prime}$ - peak systolic tricuspid annular velocity, sPAP - estimated systolic pulmonary artery pressure

\section{Tab. 3. Baseline ECG parameters.}

\begin{tabular}{lccc}
\hline & $\begin{array}{c}\text { HFREF } \\
(\mathrm{n}=46)\end{array}$ & $\begin{array}{c}\text { HFPEF } \\
(\mathrm{n}=63)\end{array}$ & $\mathrm{p}$ \\
\hline Heart rate, $/ \mathrm{min}$ & $97.7 \pm 23.7$ & $84.3 \pm 25.7$ & $<0.01$ \\
PQ interval, ms & $170(160-200)$ & $160(160-200)$ & $\mathrm{NS}$ \\
QRS duration, ms & $100(80-120)$ & $80(80-115)$ & 0.06 \\
QTc interval, ms & $452 \pm 58$ & $435 \pm 44$ & 0.06 \\
Atrial fibrillation, $\%$ & 26.7 & 46.0 & $<0.05$ \\
\hline
\end{tabular}

HFPEF - heart failure with preserved ejection fraction, HFREF - heart failure with reduced ejection fraction, NS - non-significant

$(130(110-140)$ vs $130(120-140), \mathrm{p}=\mathrm{NS})$, respectively. There was non significant difference between both groups in other parameters, i.e. body mass index, prevalence of arterial hypertension, diabetes mellitus and coronary artery disease.

The baseline echocardiographic parameters are in the Table 2. Eccentric LV hypertrophy was more prevalent in HFREF (13.1\% vs $59.1 \%, \mathrm{p}<0.0001)$ and concentric LV hypertrophy in HFPEF (50.8 \% vs $15.9 \%, p=0.0001)$. The prevalence of preserved RV systolic function after 2 years was non-significantly different between HFPEF and HFREF (65.9\% vs $57.1 \%$, p=NS). After 2 years, the mean S' was non-significantly different in HFPEF vs HFREF $(10.8 \pm 2.8 \mathrm{~cm} / \mathrm{s}$ vs $11.6 \pm 3.6 \mathrm{~cm} / \mathrm{s}, \mathrm{p}=\mathrm{NS})$.

The adequately measurable Doppler signal of tricuspid regurgitation was present in $44.4 \%$ of the patients with HFPEF vs $52.2 \%$ in HFREF $(p=N S)$. The prevalence of increased sPAP $>35 \mathrm{mmHg}$ was similar in patients with HFPEF and HFREF with an adequately measurable jet of tricuspid regurgitation $(89.3 \%$ vs $79.2 \%, p=N S$ ). We observed a weak correlation between $\mathrm{S}^{\prime}$ and SPAP in HFPEF but not in HFREF $(\mathrm{r}=-0.4, \mathrm{p}=0.005$ and $\mathrm{r}=-0.2, \mathrm{p}=\mathrm{NS})$.

LVEF was $56.8 \%$ vs $38.9 \%$, p $<0.0001$ in the 2 -year follow-up in HFPEF vs HFREF, respectively. In the surviving patients with 
Tab. 4 Selected correlations of left ventricular diastolic function parameters.

\begin{tabular}{lcc}
\hline & $\begin{array}{c}\text { HFREF } \\
(\mathrm{n}=46)\end{array}$ & $\begin{array}{c}\text { HFPEF } \\
(\mathrm{n}=63)\end{array}$ \\
\hline QTc vs. diastolic dysfunction grade & $\mathrm{NS}$ & $\mathrm{r}=0.3, \mathrm{p}<0.05$ \\
QTc vs. E & $\mathrm{NS}$ & $\mathrm{r}=0.3, \mathrm{p}<0.05$ \\
QTc vs. E' & $\mathrm{r}=-0.4, \mathrm{p}<0.05$ & $\mathrm{NS}$ \\
QTc vs. E/E' & $\mathrm{NS}$ & $\mathrm{NS}$ \\
QTc vs. DCT & $\mathrm{NS}$ & $\mathrm{r}=-0.3, \mathrm{p}<0.05$ \\
QTc vs. QRS & $\mathrm{r}=0.5, \mathrm{p}<0.01$ & $\mathrm{r}=0.3, \mathrm{p}=0.01$ \\
Diastolic dysfunction grade vs. E/E & $\mathrm{r}=0.4, \mathrm{p}<0.05$ & $\mathrm{r}=0.5, \mathrm{p}<0.0001$ \\
Diastolic dysfunction grade vs. DCT & $\mathrm{r}=-0.6, \mathrm{p}<0.001$ & $\mathrm{r}=-0.4, \mathrm{p}<0.001$ \\
Diastolic dysfunction grade vs. E & $\mathrm{r}=0.5, \mathrm{p}<0.01$ & $\mathrm{r}=0.6, \mathrm{p}<0.0001$ \\
\hline DCT - mitral wave E deceleration time, E- peak early mitral filling velocity, $\mathrm{E}^{\prime}-\mathrm{peak}$ \\
early diastolic mitral annular velocity, HFPEF - heart failure with preserved ejection \\
fraction, HFREF - heart failure with reduced ejection fraction, $\mathrm{NS}-$ non-significant
\end{tabular}

Tab. 5. Baseline laboratory parameters.

\begin{tabular}{lccc}
\hline & $\begin{array}{c}\text { HFREF } \\
(\mathrm{n}=46)\end{array}$ & $\begin{array}{c}\text { HFPEF } \\
(\mathrm{n}=63)\end{array}$ & $\mathrm{p}$ \\
\hline Serum sodium, mmol/l & $139.0 \pm 6.4$ & $139.0 \pm 3.9$ & $\mathrm{NS}$ \\
Serum potassium, mmol/1 & $4.41 \pm 0.52$ & $4.53 \pm 0.60$ & $\mathrm{NS}$ \\
Glycaemia, $\mathrm{mmol} / \mathrm{l}$ & $6.30(5.38-7.80)$ & $5.95(5.38-8.10)$ & $\mathrm{NS}$ \\
NT-proBNP, $\mathrm{ng} / \mathrm{ml}$ & $5467(3428-10368)$ & $3006(1734-6016)$ & $<0.01$ \\
Creatinine, umol/1 & $98(80-122)$ & $102(82-156)$ & $\mathrm{NS}$ \\
Glomerular filtration, $\mathrm{ml} / \mathrm{s}$ & $1.08 \pm 0.37$ & $0.93 \pm 0.47$ & $\mathrm{NS}$ \\
Hemoglobin, $\mathrm{g} / \mathrm{l}$ & $135 \pm 20$ & $123 \pm 21$ & $<0.01$ \\
\hline
\end{tabular}

HFPEF - heart failure with preserved ejection fraction, HFREF - heart failure with reduced ejection fraction, NS - non-significant

Tab. 6. Treatment on admission.

\begin{tabular}{lccc}
\hline & $\begin{array}{c}\text { HFREF } \\
(\mathrm{n}=46)\end{array}$ & $\begin{array}{c}\text { HFPEF } \\
(\mathrm{n}=63)\end{array}$ & $\mathrm{p}$ \\
\hline Diuretics, \% & 64.4 & 73.0 & $\mathrm{NS}$ \\
ACE inhibitors/ARB, \% & 60.9 & 77.8 & 0.056 \\
Betablockers, \% & 62.2 & 71.4 & $\mathrm{NS}$ \\
MRA, \% & 40.0 & 20.6 & $<0.05$ \\
Digitalis, \% & 33.3 & 27.0 & $\mathrm{NS}$ \\
ICD/CRT, \% & 13.3 & 1.6 & $<0.05$ \\
PCI/CABG, \% & 34.8 & 20.6 & $\mathrm{NS}$ \\
Antiplatelet therapy, \% & 43.5 & 48.4 & $\mathrm{NS}$ \\
Anticoagulants, \% & 23.9 & 28.6 & $\mathrm{NS}$ \\
Statins, \% & 30.4 & 31.7 & $\mathrm{NS}$ \\
\hline
\end{tabular}

ACE - angiotensin-converting enzyme, ARB - angiotensin II receptor antagonists, CABG - coronary artery bypass grafting, CRT - cardiac resynchronization therapy, HFPEF - heart failure with preserved ejection fraction, HFREF - heart failure with reduced ejection fraction, ICD - implantable cardioverter-defibrillator, MRA - mineralocorticoid receptor antagonists, NS - non-significant

HFREF, the LVEF improved from the mean $27 \%$ to $39 \%$, while the LVEF in HFPEF retained almost the same. LV end-diastolic diameter (LVEDD) and LV mass index (LVMI) improved in 2-years in HFPEF vs HFREF $(49.0 \pm 7.4 \mathrm{~mm}$ vs $59.9 \pm 9.2 \mathrm{~mm}, \mathrm{p}<0.001$ and $\left.124.4 \pm 50.2 \mathrm{~g} / \mathrm{m}^{2} \mathrm{vs} 151 \pm 64.6 \mathrm{~g} / \mathrm{m}^{2}, \mathrm{p}=0.06\right)$.

The ECG parameters on admission are in the Table 3. The heart rate was better controlled in both groups in the final checkup visit (HFPEF vs HFREF: $74 \pm 18 / \mathrm{min}$ vs $79 \pm 18 / \mathrm{min}, \mathrm{p}=\mathrm{NS}$ ). The QTc interval after 2 years in HFPEF vs HFREF was $431 \pm 44 \mathrm{~ms}$ vs $439 \pm 48 \mathrm{~ms}(\mathrm{p}=\mathrm{NS})$. We correlated the echocardiographic parameters of diastolic dysfunction with QTc and QRS. The results are in the Table 4. In HFPEF and HFREF, we did not find any significant correlation between QRS and E/E', QRS and E, QRS and the diastolic dysfunction degree.

The laboratory parameters on admission are in Table 5. After 24 months, the N-terminal-pro-brain natriuretic peptide (NTproBNP) as well as hemoglobin were non-significantly different in HFPEF vs HFREF (median (IQR) $1335 \mathrm{ng} / \mathrm{ml}$ (966-2229) vs $1178 \mathrm{ng} / \mathrm{ml}(346-2546), \mathrm{p}=\mathrm{NS})$ and $(129 \mathrm{~g} / \mathrm{l} \pm 19 \mathrm{vs} 133 \mathrm{~g} / \mathrm{l} \pm 18$, $\mathrm{p}=\mathrm{NS})$. Although the level of NT-proBNP was much lower in both groups compared to the baseline values, the reduction was more prominent in HFREF. Hemoglobin in HFPEF slightly improved compared to initial hospitalization.

The treatment on admission is in Table 6. In the 2-year followup, the usage of angiotensin converting enzyme (ACE) inhibitors/ angiotensin receptor blockers (ARB) was non-significantly different in HFPEF vs HFREF (76.6 \% vs $86.7 \%$, $\mathrm{p}=\mathrm{NS})$. The prescription of mineralocorticoid receptor antagonists (MRA) was non-significantly higher in HFREF vs HFPEF after 2 years (46.7\% vs $40.4 \%, p=N S)$, but it was much higher in the HFPEF group compared to the baseline.

ICD/CRT implantation and coronary revascularization (CABG/PCI) after 2 years were performed more often in patients with HFREF vs HFPEF (50 \% vs $19 \%$, p $<0.001$ and $54.4 \%$ vs $23.8 \%, p=0.001)$. The usage of anticoagulants $(42.2 \%$ vs $30.0 \%, \mathrm{p}=\mathrm{NS})$, antiplatelet therapy ( $42.2 \%$ vs $63.3 \%, \mathrm{p}=\mathrm{NS})$ and statins ( $40.0 \%$ vs $58.6 \%, \mathrm{p}=\mathrm{NS}$ ) was non-significantly different after 2 years in HFPEF vs HFREF, but it was higher compared to the baseline.

The final follow-up visits were done 24 months after the initial enrollment. During the 2-year follow-up, there were 18 deaths in the HFPEF group and 17 deaths in the HFREF group (28.6\% vs $37.0 \%, \mathrm{p}=\mathrm{NS}$ ). The CV cause of death was found in $11 \mathrm{HFPEF}$ patients and in 10 HFREF patients $(17.5 \%$ vs $21.7 \%$, p =NS). Kaplan-Meier curves of all-cause and CV mortality free survival are shown in the Figures 1 and 2.

In HFPEF, neither medical therapy (diuretics, betablockers, MRA, antiplatelet therapy, statins and ACE inhibitors/ARB) nor ICD/CRT and PCI/CABG influenced all-cause mortality. Only patients treated with anticoagulants had a significantly lower all-

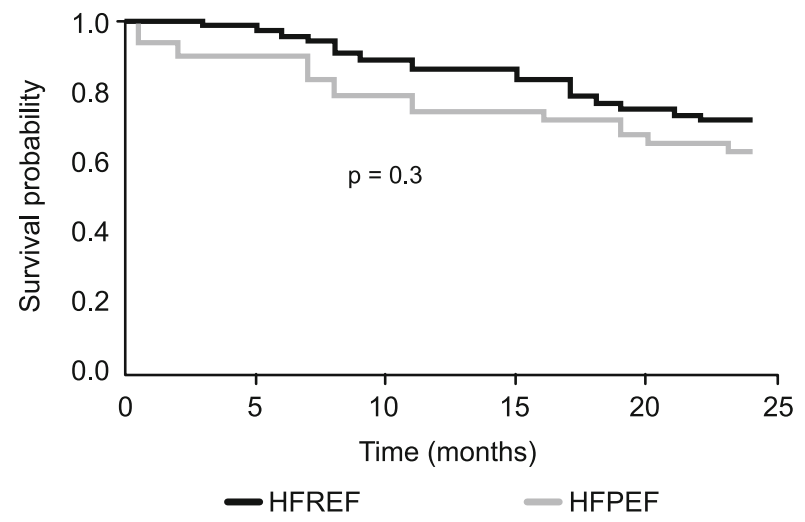

Fig. 1. All-cause mortality in HFPEF vs HFREF in 2-year follow-up. 
407-412

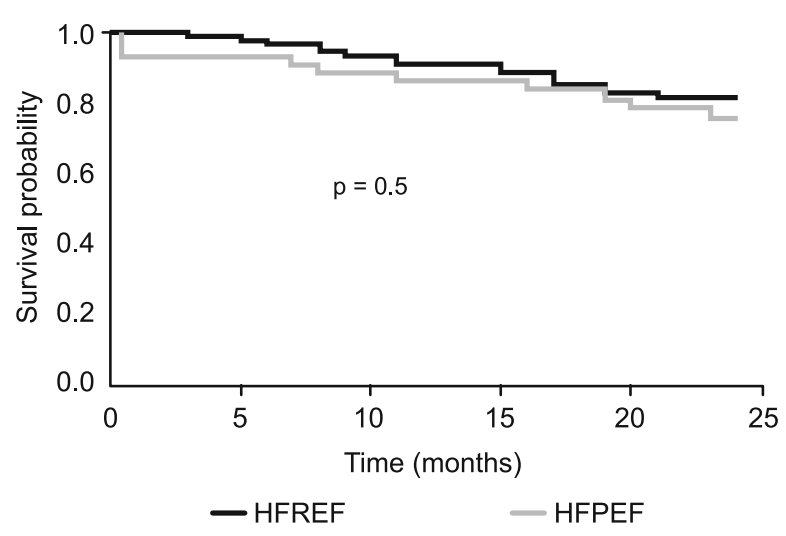

Fig. 2. Cardiovascular mortality in HFPEF vs HFREF in 2-year follow-up.

cause mortality compared to those not treated ( $12.0 \%$ vs $39.5 \%$, $\mathrm{p}=0.02$ ). In HFREF, we observed a significantly lower all-cause mortality in patients treated with betablockers compared to those not treated $(25 \%$ vs $80 \%, \mathrm{p}<0.001)$ as well as in patients treated with ACE inhibitors/ARB ( $28.2 \%$ vs $85.7 \%, \mathrm{p}<0.001)$. Otherwise, there was no difference in mortality under other treatments (diuretics, MRA, antiplatelet therapy, anticoagulants, ICD/CRT and $\mathrm{PCI} / \mathrm{CABG})$.

Compared to the patients with dysfunctional RV, the patients with preserved RV systolic function in HFPEF had a significantly better 2-year survival $(76.6 \%$ vs $56.3 \%, \mathrm{p}=0.045)$. The same trend was seen in HFREF and preserved RV function compared to dysfunctional RV (73.7 \% vs $55.6 \%$, p=NS). The patients with HFPEF who died had a strong trend to worse $S^{\prime}(11.9 \pm 3.4 \mathrm{~cm} / \mathrm{s}$ vs $13.6 \pm 3.1 \mathrm{~cm} / \mathrm{s}, \mathrm{p}=0.055)$ and prolonged QTc interval $(454 \pm 42 \mathrm{~ms}$ vs $427 \pm 42 \mathrm{~ms}, \mathrm{p}=0.058)$. The patients with HFREF who died also had a strong trend to worse $\mathrm{S}^{\prime}(9.4 \pm 3.1 \mathrm{~cm} / \mathrm{s}$ vs $11.5 \pm 3.1$ $\mathrm{cm} / \mathrm{s}, \mathrm{p}=0.068)$ and a lower level of hemoglobin $(124.3 \pm 24 \mathrm{~g} / \mathrm{l}$ vs $140.4 \pm 15 \mathrm{~g} / \mathrm{l}, \mathrm{p}=0.01)$.

There was a non-significant trend toward a higher prevalence of cumulative CV endpoint in HFREF vs HFPEF (41.3\% vs $31.7 \%$, $\mathrm{p}=\mathrm{NS}$ ). Re-hospitalizations for HF were non-significantly different in HFPEF and HFREF in the 2-year follow-up (46.0 \% vs $50.0 \%, \mathrm{p}=\mathrm{NS}$ ).

Using a Cox model after performing the univariate analysis, the QTc interval and the preserved RV systolic function emerged as an independent predictor of all-cause mortality in patients with HFPEF (HR 1.7 per 40 ms change, $95 \%$ CI 1.1-2.6, $\mathrm{p}=0.02$ and HR $0.38,95 \%$ CI 0.15 to $0.93, p=0.03$ ).

\section{Discussion}

The present prospective study of 109 patients, focused on HFPEF, with a 24-month follow-up, has three main prognostic findings: 1) the patients with HFPEF had a non-significant trend to better survival and a non-significant trend to lower CV mortality compared to HFREF. 2) Anticoagulants were the only therapy that lowered mortality in HFPEF. 3) The preserved RV systolic function and the QTc interval length merged as the independent predictors of a 2-year survival.

The mortality burden of HFPEF is substantial, ranging from $10 \%-30 \%$ annually and long-term mortality in HFPEF vs HFREF is comparable $(2,9,10)$. Although in-hospital mortality is slightly higher in HFREF, 30-day to 1 year mortality is already similar between both groups $(2,6,9)$. We observed a non-significantly better survival in patients with HFPEF vs HFREF in the 2-year follow-up and similar findings were in CV mortality. Similarly to another study, $61 \%$ of the deceased HFPEF patients died from $\mathrm{CV}$ causes, which was non-significantly different compared to $65 \%$ in HFREF (15). After the 2-year follow-up, both HF groups, seem to have almost the same prevalence of comorbidities (arterial hypertension, diabetes mellitus, renal dysfunction), except for the prevalence of atrial fibrillation, which was higher in HFPEF. The cumulative CV endpoint was non-significantly different in HFREF vs HFPEF and the rate of hospitalization for HF in 2 years was similar, too. A trend to worse prognosis in terms of all above-mentioned parameters (all-cause mortality, CV mortality and cumulative CV endpoint) was found in HFREF.

During the follow-up, we observed that no other treatment than anticoagulants significantly reduced all-cause mortality in HFPEF ( $12.0 \%$ vs $39.5 \%, p=0.02)$. We did not distinguish between oral anticoagulants, namely warfarin, and novel anticoagulants (NOAC), because at the beginning of the study, NOAC were not broadly used in our country. The main proportion of the anticoagulation therapy was used due to atrial fibrillation. At the end of the study, there were $78 \%$ vs $48 \%$ patients in HFPEF vs HFREF $(p=0.012)$ with the history of atrial fibrillation. According to these data, the patients in both groups were strongly undertreated. We expect that the risk profile in our HFPEF patients favors the anticoagulation therapy because of older age, a high prevalence of arterial hypertension and female gender. The low proportion of the treated patients might be partially explained by a higher bleeding risk, while HFPEF patients generally have a poor risk profile. However, even though there is a more profound mismatch in the prevalence of atrial fibrillation and anticoagulation treatment, we did not observe a significant difference in the prevalence of stroke in both groups. In our study, the patients with HFPEF treated with anticoagulants had significantly lower all-cause mortality vs those not treated. This was not true for HFREF. Shore et al. also observed that HFPEF patients on chronic anticoagulation for a concurrent indication were noted to live longer than those not treated (16). In this study, the outcome was not linked to anticoagulation solely due to atrial fibrillation, because no significant difference in mortality was seen in patients with atrial fibrillation on chronic anticoagulation compared to those not treated (16). Moreover, no statistically significant difference in the stroke risk was observed between the patients with atrial fibrillation and HFPEF vs HFREF (17). Thus, the mechanism of a better overall impact of anticoagulation on mortality in HFPEF is unknown.

In accordance with the published studies, we did not find any favorable influence of the medications broadly recommended in HFREF on the survival in HFPEF (11). Significantly lower allcause mortality was noted in patients with HFREF treated with 
betablockers and ACE inhibitors/ARB compared to those not treated. Interestingly, all-cause mortality was not influenced by the MRA treatment in HFREF.

We identified the QTc interval and the RV systolic function as independent predictors of a 2-year survival in HFPEF. We tried to find the link between the QTc and the diastolic function of the LV, based on the assumption that the QTc interval encompasses both the depolarization (systole) and repolarization (diastole) phase. We assume that the repolarization phase is responsible for the influence on the predictive value of QTc in HFPEF, hence it shows a worse diastolic dysfunction grade. A few studies found the correlation between QTc and the parameters of diastolic dysfunction $(18,19)$. The authors hypothesized that in the narrow QRS, the association between QTc and $\mathrm{E}^{\prime}$ appeared to be a function of a prolonged repolarization, otherwise in the wide QRS it might be influenced by the LV intraventricular and interventricular dyssynchrony (19). We assessed that the QTc interval in HFPEF correlates significantly with the diastolic dysfunction grade, E velocity and DCT but not with the $E / E^{\prime}$ ratio and $E^{\prime}$. QTc interval did correlate well with the QRS duration, which could ascertain the above-mentioned hypothesis of Wilcox et al (19). The most powerful LV diastolic parameter is in our opinion solely the diastolic dysfunction grade, because it merges several echocardiographic parameters. We hypothesize that the influence of other parameters, especially the $\mathrm{E} / \mathrm{E}^{\prime}$, might be distorted by the high prevalence of atrial fibrillation and the problematic assessment of the $\mathrm{E} / \mathrm{E}^{\prime}$ ratio under this condition (20). We think that QTc might be a useful parameter to predict diastolic dysfunction and, according to our data, QTc merges as an independent predictor of a 2-year survival, too.

The second independent predictor of a 2-year survival in HFPEF was RV systolic function. We observed that preserved RV systolic function was significantly more common in HFPEF and the mean S' was significantly higher in HFPEF vs HFREF. The dysfunctional RV in HFPEF is present in around $1 / 3$ of the patients depending on the study design $(21,22)$. Several techniques were used to assess the RV function $(13,22,23)$. We evaluated the RV systolic function by S' because RV volume measurements and tricuspid annular plane systolic excursion (TAPSE) might not be adequately obtainable in all patients, especially in those with decreased echogenicity. The best-known mechanism of RV systolic dysfunction is the passive transmission of LV filling pressure, reactive vasoconstriction and remodeling of pulmonary arteries leading to postcapillary pulmonary hypertension. In our study, the RV systolic function seemed to deteriorate with the increasing severity of pulmonary hypertension. This was not the case in HFREF. The correlation between the estimated SPAP and S' may be negatively influenced by the fact that the dysfunctional RV itself might cause the tricuspid gradient to be lower and hence sPAP could not be adequately assessed. Nevertheless, not solely pulmonary hypertension causes the RV dysfunction. Septal dysfunction related to the LV diastolic dysfunction leads to a decrease of the septal role in the RV ejection and thus causes a systolic mechanical dyssynchrony of the RV (24). There is only limited evidence about the prognostic importance of RV systolic dysfunction in HFPEF (21, $25,26)$. Irrespective of the mechanism of the RV dysfunction and the examination technique, the RV systolic dysfunction is an independent predictor of a poor prognosis in patients with HF in general $(13,23-25)$. We observed in our study that the preserved $\mathrm{RV}$ systolic function was an independent predictor of a better survival in HFPEF.

\section{Conclusion}

We observed that patients with HFPEF had a non-significant trend to better survival and lower CV mortality compared to HFREF. Anticoagulants were the only therapy that significantly lowered mortality in HFPEF. The preserved RV systolic function and QTc interval length merged as the independent predictors of a 2-year survival.

\section{Learning points}

All-cause and CV mortality were non-significantly lower in HFPEF compared to HFREF in a mid-term follow-up.

RV systolic dysfunction assessed by tissue Doppler imaging and QTc interval might predict HFPEF patients with adverse outcome.

Anticoagulants might lower mortality in HFPEF, but the mechanism is unknown.

\section{References}

1. Kitzman DW, Gardin JM, Gottdiener JS, Arnold A, Boineau R, Aurigemma G et al. Importance of heart failure with preserved systolic function in patients $>$ or $=65$ years of age. CHS Research Group. Cardiovascular Health Study. Am J Cardiol 2001; 87: 413-419.

2. Bhatia RS, Tu JV, Lee D, Austin PC, Fang J, Haouzi A et al. Outcome of heart failure with preserved ejection fraction in a population-based study. N Engl J Med 2006; 355: 260-269.

3. Yusuf S, Pfeffer MA, Swedberg K, Granger CB, Held P, Mcmurray JJ et al. Effects of candesartan in patients with chronic heart failure and preserved left-ventricular ejection fraction: The CHARM-Preserve Trial. Lancet 2003; 362: 777-781.

4. Ahmed A, Rich MW, Fleg JL, Zile MR, Young JB, Kitzman DW et al. Effects of digoxin on morbidity and mortality in diastolic heart failure: The ancillary Digitalis Investigation Group trial. Circulation 2006; 114: $397-403$.

5. Campbell RT, Jhund PS, Castagno D, Hawkins NM, Petrie MC, Mcmurray JJ. What have we learned about patients with heart failure and preserved ejection fraction from DIG-PEF, CHARM-preserved, and I-PRESERVE? J Am CollCardiol 2012; 60: 2349-2356.

6. Meta-analysis Global Group in Chronic Heart Failure (MAGGIC). The survival of patients with heart failure with preserved or reduced left ventricular ejection fraction: an individual patient data meta-analysis. Eur Heart J 2012; 33: 1750-1757.

7. Burkhoff D. Mortality in heart failure with preserved ejection fraction: an unacceptably high rate. Eur Heart J 2012; 33: 1718-1720.

8. Brouwers FP, de Boer RA, van der Harst P, Voors AA, Gansevoort RT, Bakker SJ et al. Incidence and epidemiology of new onset heart failure with preserved vs reduced ejection fraction in a community-based cohort: 11-year follow-up of PREVEND. Eur Heart J 2013; 34: 1424-1431. 
407-412

9. Fonarow GC, Stough WG, Abraham WT, Albert NM, Gheorghiade M, Greenberg BH et al. Characteristics, treatments, and outcomes of patients with preserved systolic function hospitalized for heart failure: a report from the OPTIMIZE-HF Registry. J Am Coll Cardiol 2007; 50: 768-777.

10. Zile MR, Gaasch WH, Anand IS, Haass M, Little WC, Miller AB et al. Mode of death in patients with heart failure and a preserved ejection fraction: results from the Irbesartan in Heart Failure With Preserved Ejection Fraction Study (I-Preserve) trial. Circulation 2010; 121: $1393-1405$.

11. Mcmurray JJV, Adamopoulos S, Anker SD, Auricchio A, Böhm $\mathbf{M}$, Dickstein $\mathbf{K}$ et al. ESC Guidelines for the diagnosis and treatment of acute and chronic heart failure 2012: The Task Force for the Diagnosis and Treatment of Acute and Chronic Heart Failure 2012 of the European Society of Cardiology. Developed in collaboration with the Heart Failure Association (HFA) of the ESC Eur Heart J 2012; 33: 1787-847.

12. Douglas PS, Garcia MJ, Haines DE, Lai WW, Manning WJ, Patel AR et al. ACCF/ASE/AHA/ASNC/HFSA/HRS/SCAI/SC CM/SCCT/S CMR 2011 Appropriate Use Criteria for Echocardiography. A Report of the American College of Cardiology Foundation Appropriate Use Criteria Task Force; American Society of Echocardiography; American Heart Association; American Society of Nuclear Cardiology; Heart Failure Society of America; Heart Rhythm Society; Society for Cardiovascular Angiography and Interventions; Society of Critical Care Medicine; Society of Cardiovascular Computed Tomography; Society for Cardiovascular Magnetic Resonance; American College of Chest Physicians. J Am SocEchocardiogr 2011; 24: 229-267.

13. Meluzin J, Spinarova L, Dusek L, Toman J, Hude P, Krejci J. Prognostic importance of the right ventricular function assessed by Doppler tissue imaging. Eur J Echocardiography 2003; 4: 262-271.

14. Rudski LG, Lai WW, Afilalo J, Hua L, Handschumacher MD, Chandrasekaran K et al. Guidelines for the Echocardiographic Assessment of the Right Heart in Adults: A Report from the American Society of Echocardiography Endorsed by the European Association of Echocardiography, a registered branch of the European Society of Cardiology, and the Canadian Society of Echocardiography. J Am SocEchocardiogr 2010; 23: 685-713.

15. Chan MMY, Lam CSP. How do patients with heart failure with preserved ejection fraction die? Eur J Heart fail 2013; 15: 604-613.
16. Shore S, Aggarwal V, Zolty R. Chronic anticoagulation and long-term mortality in patients presenting for heart failure with preserved ejection fraction (HFPEF). J Am Coll Cardiol 2012; 59 (13a1): 1038-1038.

17. Banerjee A, Taillandier S, Olesen JB, Lane DA, Lallemand B, Lip GY et al. Ejection fraction and outcomes in patients with atrial fibrillation and heart failure: the Loire Valley Atrial Fibrillation Project. Eur J Heart Fail 2012; 14: 295-301.

18. Mayet J, Shahi M, McGrath K, Poulter NR, Sever PS, Foale RA et al. Left ventricular hypertrophy and QT dispersion in hypertension. Hypertension 1996; 28 (5): 791-796.

19. Wilcox JE, Rosenberg J, Vallakati A, Gheorghiade M, Shah SJ. Usefulness of Electrocardiographic QT Interval to Predict Left Ventricular Diastolic Dysfunction. Am J Cardiol 2011; 108: 1760-1766.

20. Nagueh SF, Appleton CP, Gillebert TC, Marino PN, Oh JK, Smiseth OA et al. Recommendations for the evaluation of left ventricular diastolic function by echocardiography. Eur J Echocardiogr 2009; 10: 165-193.

21. Melenovsky V, Hwang SJ, Lin G, Redfield MM, Borlaug BA. Right heart dysfunction in heart failure with preserved ejection fraction. Eur Heart J 2014; 35: 3452-3462.

22. Puwanant S, Priester TC, Mookadam F, Bruce CJ, Redfield MM, Chandrasekaran K. Right ventricular function in patients with preserved and reduced ejection fraction heart failure. Eur J Echocardiogr 2009; 10: 733-737.

23. Damy T, Viallet C, Lairez O, Deswarte G, Paulino A, Maison P et al. Comparison of four right ventricular systolic echocardiographic parameters to predict adverse outcomes in chronic heart failure. Eur J Heart Fail 2009; 11: 818-24.

24. Luo C, Ramachandran D, Ware D, Ma TS, Clark JW. Modeling left ventricular diastolic dysfunction: classification and key indicators. Theor Biol Med Model 2011; 8: 14.

25. Damy T, Kallvikbacka-Bennett A, Goode K, Khaleva O, Lewinter C, Hobkirk J et al. Prevalence of, associations with, and prognostic value of tricuspid annular plane systolic excursion (TAPSE) among out-patients referred for the evaluation of heart failure. J Card Fail 2012; 18: 216-225.

26. Cenkerova K, Dubrava J, Pokorna V, Kaluzay J, Jurkovicova O. Right ventricular systolic dysfunction and its prognostic value in heart failure with preserved ejection fraction. Acta Cardiol 2015; 70: 387-393.

Received December 31, 2015. Accepted January 15, 2016. 